Article

\title{
Experimental Study on Hydrate Formation and Flow Characteristics with High Water Cuts
}

\author{
Shidong Zhou * $\mathbb{D}$, Xiaokang Chen, Chengyuan He, Shuli Wang, Shuhua Zhao and Xiaofang Lv \\ Jiangsu Key Laboratory of Oil and Gas Storage and Transportation Technology, Changzhou University, \\ Changzhou 213164, China; chenxk7215@126.com (X.C.); fwsa369704@163.com (C.H.); ws1@cczu.edu.cn (S.W.); \\ zsh@cczu.edu.cn (S.Z.); lvxiaofang5@cczu.edu.cn (X.L.) \\ * Correspondence: zsd@cczu.edu.cn
}

Received: 20 August 2018; Accepted: 29 September 2018; Published: 30 September 2018

\begin{abstract}
The rapid increasing amount of produced water in the deep-water and ultra-deep-water fields, especially those at their later development stages, increases the risk of the occurrence of hydrates plugging. In order to prevent and remediate the hydrates risks, it is important to understand the hydrate formation, slurry flow, and plugging characteristics and mechanisms under high water cuts conditions. In this paper, experiments with high water cuts ranging from $60-100 \%$ were conducted using a high pressure flow loop with observation windows. The whole processes of the hydrate formation, slurry flow and plugging is visually seen and recorded, and has been discussed and explained in detail in this paper. Moreover, it is found that the increasing water cuts shorten the induction time, but increase the volume fraction of hydrates. As the water cuts increase, the flow time of the hydrate slurry decreases, which serves as a critical parameter for the safe operation of the pipeline. In addition, different hypothetical mechanisms have been proposed for the medium and high water cuts conditions. We believe that this research can provide theoretical support for the safe transportation of oil and gas development in the high water cut oilfield.
\end{abstract}

Keywords: high water cut; hydrates; formation; plugging; mechanisms

\section{Introduction}

Flow assurance problems such as hydrates [1,2], wax deposition [3-7], and asphaltenes are critical operating challenges to ensure the safe and efficient transportation of unprocessed well steams especially in offshore oil fields [8,9]. As the offshore deep-water reservoirs mature, especially if secondary or tertiary recovery methods are employed, the amount of produced water climbs, which increases the risk of gas hydrate plugging the pipelines. Understanding the hydrate plugging mechanism in high water cut systems is of great significance for the safe operation of offshore multiphase pipelines.

It has been well-known that gas hydrates are ice-like solids, where the light hydrocarbon species (e.g., methane) get trapped in the hydrogen bonded molecular cage of water under high pressure and low temperature conditions [10]. In the past two decades, the community has focused on different techniques to control hydrate risk, such as adding inhibitors, applying cold flow, all of those have been tested in both laboratory and pilot scales [11]. However, limited attention has been paid to the plugging mechanisms and the flow characteristics of hydrate slurry. Zerpa et al. [12] proposed a multiphase flow model for hydrate slurry, which can effectively predict hydrate formation rate and formation temperature. Wang et al. [13] simulated the submarine pipeline transportation process using natural gas hydrate formation and slurry flow device, and analyzed the morphological changes during the formation of hydrate. Hydrates shapes will undergo changes in the form of emulsions, granules, pellets and clouds. Lorenzo et al. $[14,15]$ reported the first pilot-scale experimental data for hydrate 
blockage formation in a gas-dominant single-pass flow loop, which demonstrated hydrate blockage rates were similar to what has been reported in industrial cases [16]. Chen et al. [17] used a flow loop to study the flow characteristics of natural gas-water-diesel hydrate formation at $5-80 \%$ moisture and explored the plugging mechanism. Akhfash et al. [18] conducted an experimental study on hydrate blockage in oil-water dispersion systems through a high-pressure sapphire visual reaction kettle. The results demonstrated that a water cut of less than $30 \%$ in the dispersion system did not increase the hydrate growth rate or cause plug; when the water cut increased to the range of $50 \%$ and $70 \%$, the hydrate will severely block the pipeline [19]. Song et al. [20] studied the plug characteristics of the natural gas-diesel-water system using a high pressure flow loop. The results showed that the plug was mainly caused by the formation and growth of the hydrate deposition layer. The mechanisms of rapid plug and gradual plug were proposed. In addition, the sludge-like hydrate, the flocculated hydrate deposit layer, and the phenomenon during the plug process were observed through the window.

Even though some attention has been given to the hydrates plug characteristics, there are very limited studies that focus on the medium and high aqueous systems. The application of $\mathrm{CO}_{2}$ hydrate in following areas has a great effect. Firstly, in many countries, high $\mathrm{CO}_{2}$-containing oil and gas fields have been found, of which $\mathrm{CO}_{2}$ content exceeds $0.7 \mathrm{~mol}$ [21]. Secondly, $\mathrm{CO}_{2}$ hydrate is widely used in the field of cool storage, carbon dioxide complementation, and submarine sealing $\mathrm{CO}_{2}$.Therefore, it is instructive to study $\mathrm{CO}_{2}$ hydrate for safe transportation of deep-sea oil and gas, preventing hydrate plugging. In the present work, a high-pressure flow loop with two transparent sapphire windows was newly constructed. The hydrates formation and plug characteristics with $60-100 \%$ water cuts were investigated and explained in detail. Moreover, the effect of water cuts on the induction time and volume fraction of hydrate formation were studied. The effect of water cuts on the time of hydrate slurry flows, friction change, and differential pressure change were reported as well. These results are believed to provide theoretical support for the safe transportation of oil and gas development in the high water cut oilfield.

\section{Experimental Program}

\subsection{Experimental Facility}

A high-pressure flow loop is newly constructed in Jiangsu Key Laboratory of Oil and Gas Storage and Transportation at Changzhou University (Figure 1). It consists of gas intake unit, liquid inlet unit, refrigeration system, visual reaction kettle, pipeline test section and a data acquisition system. The test section consists of five straight pipe sections with lengths of $1 \mathrm{~m}, 3 \mathrm{~m}, 2.5 \mathrm{~m}, 1 \mathrm{~m}, 0.5 \mathrm{~m}$, respectively, a semi-circular pipe section with a radius of $0.63 \mathrm{~m}$, and a partially connected high-pressure hose with a total length of $15 \mathrm{~m}$. The inner diameter of the pipeline is $2.54 \mathrm{~cm}$, and the design pressure of the pipeline is $15 \mathrm{MPa}$. The total volume of the pipeline is $10 \mathrm{~L}$. The temperature is controlled by the refrigerator through the water bath. The control temperature ranges from -15 to $20{ }^{\circ} \mathrm{C}$ with an accuracy of $\pm 1{ }^{\circ} \mathrm{C}$. The whole unit is equipped with differential pressure sensor (Honeywell STD720), pressure transmitter (Rosemount 3051), liquid turbine flowmeter (Dalian Youke Instrument and Meter Development Center, YK-LWGY-04), gas mass flow meter (Emerson process control flow Technology Co., Ltd., Nanjing, China, CMFS010M323N2BZMCZZ), and a mass flowmeter (Dalian Youke Instrument and Meter Development Center, YKLK-S-025). All the temperature, pressure, differential pressure, and flow rate data are collected and recorded by the data acquisition system. The flow loop also has two transparent sapphire windows to observe the actual experimental phenomenon at different locations of the pipeline. The schematic diagram of the flow loop is shown in Figure 2. 


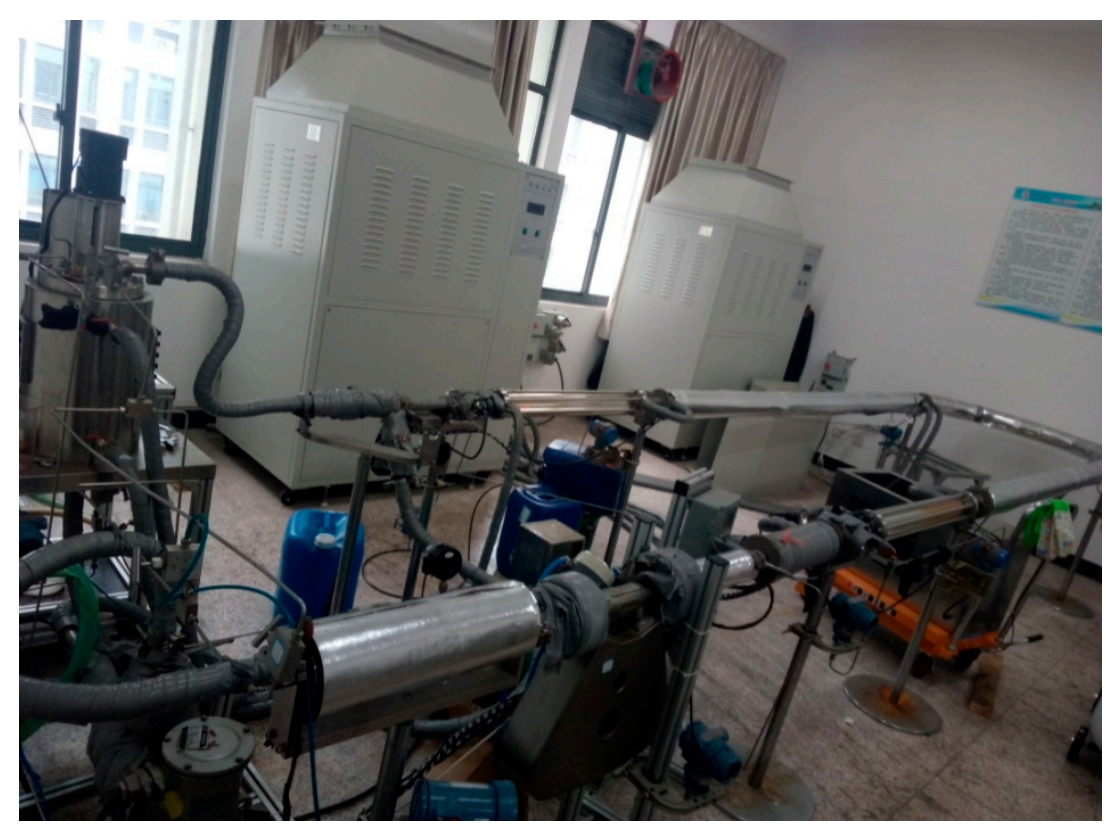

Figure 1. High-pressure flow loop at ChangZhou university.

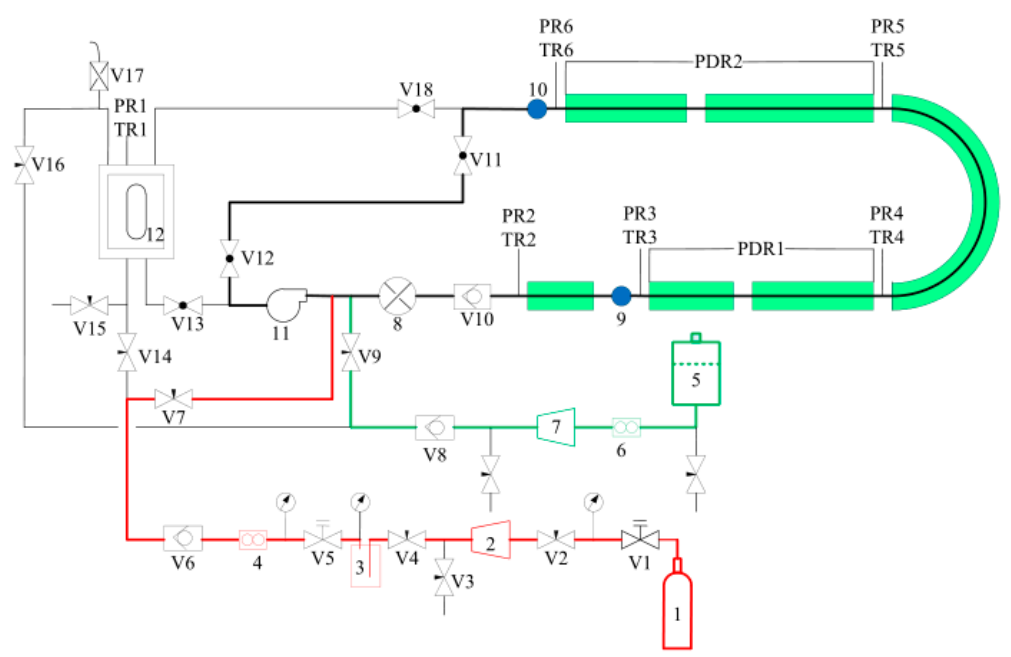

Figure 2. Schematic diagram of the High-pressure flow loop: 1-gas cylinder; 2-gas booster pump; 3-buffer tank; 4-gas mass flow meter; 5-storage tank; 6-liquid mass flow meter; 7-liquid booster pump; 8-mass flowmeter; 9,10-observation window; 11-circulation pump; 12-visual reaction kettle; V1,V5-pressure regulating valve; V2-V4,V7,V9,V14-V16,V18 - needle valve; V6,V8,V10 — check valve; V11-13,V18-high pressure ball valve; V17-safety valve; PR1-5-pressure sensor; TR1-5-temperature sensor; PDR1,PDR2-differential pressure.

\subsection{Experimental Materials}

In this work, liquid paraffin, tap water and $\mathrm{CO}_{2}$ are used to simulate the real fluids in the pipeline. Since it is relatively easy to form a hydrate with carbon dioxide and it has the same crystal structure as methane hydrate (Zhou et al., 2018), $\mathrm{CO}_{2}$ is used as the gas phase in this experiment, which is supplied by Changzhou Jinghua Industrial Gas Company (Zhou et al., 2018). The viscosity and density of liquid paraffin are $12 \mathrm{~mm}^{2} / \mathrm{s}, 860 \mathrm{Kg} / \mathrm{m}^{3}$, respectively, which is provided by Henan Hua-long Pharmaceutical Company. In this experiment, the liquid paraffin is dyed red by oil-soluble for easy observation, which is provided by Dye chemical company. 


\subsection{Operation Procedures}

The experimental procedures are given as follows: (1) The tightness of the flow loop system was checked before starting the experiments; (2) The vacuum pump was utilized to pump the gas to the pipeline until the pressure reached $0.02 \mathrm{MPa}$; (3) The data acquisition system was started; (4) The double-plunger pump was started to drive the mixture of water and oil into the pipeline until the total volume reached $9 \mathrm{~L}$ (the corresponding liquid hold rate is $90 \%$ ); (5) The cooling water bath was started and the temperature was set to the desired value; (6) The circulation pump was turned on and the pump speed was adjusted so that the water in the line circulated at the desired flow rate; (7) The intake valve was opened until the system pressure reached the experimental pressure, then the gas-liquid mixture flow is made stable for at least half an hour to achieve the dissolution balance. Until the pipeline cooled, the gas tank was opened to provide gas to the loop; (8) The hydrate formation process was observed through the visual window, and data such as pressure, temperature, flow rate, and differential pressure were collected and recorded by the data acquisition system; (9) The liquid inside the experimental system was discharged after each test. Then the pipeline was cleaned, and the compressor was purged for $1 \mathrm{~h}$.

\subsection{Test Matrix}

In this study, all the experiments were performed at a constant volume. The initial pressure is $3 \mathrm{MPa}$ and the initial temperature is $10.5^{\circ} \mathrm{C}$. In this experiment, we calculate the phase velocities, and the gas flow rate and liquid flow rate in the pipe are $0.172 \mathrm{~m} / \mathrm{s}$ and $0.893 \mathrm{~m} / \mathrm{s}$. By looking at the Lee flow diagram and experimental observations, we can determine that it is slug-flow. In addition, repeated experiments were conducted, and it has a repeatability of $10 \%$. Detailed experimental conditions are shown in Table 1.

Table 1. Experimental conditions at different water cuts.

\begin{tabular}{cccc}
\hline Case & Water Cut Rage & Water Cut (\%) & Initial Flow Rate (L/min) \\
\hline 1 & Pure water & 100 & 18.22 \\
2 & Extra high water cut & 95 & 18.23 \\
3 & High water cut & 80 & 17.22 \\
4 & Medium water cut & 60 & 15.23 \\
\hline
\end{tabular}

\section{Results and Discussion}

\subsection{Experimental Observations}

In order to explore the effect of water cuts on hydrate formation and plug, experiments with different water cuts $(60 \%, 80 \%, 95 \%, 100 \%)$ were conducted under the condition of the initial pressure of $3 \mathrm{MPa}$, the temperature of $1.5^{\circ} \mathrm{C}$ and the liquid holding capacity of $90 \%$. Figure 3 shows a typical curve on the hydrates formation and plug process, which can be divided into the following three stages.

Stage I is the stage of cooling and dissolving. In this stage, after the inlet temperature and pressure reach the dissolution equilibrium, the system will be cooled down. As the fluid temperature decreases, the pressure inside the system also decreases. As shown in Figure 3, the temperature drops to the phase equilibrium temperature point $(280.02 \mathrm{~K}, 2.8385 \mathrm{MPa})$. The specific flow pattern in the pipeline can be observed through the window. From group A in Figure 4, it can be found that the mixture of gas, oil and water flows rapidly in the pipeline. Because of the cutting effect of the pump, the fluid in the pipe will appear a partially dispersed system, which has some dissolved gas and small bubbles. It is a stable gas-liquid multiphase flow. Although the temperature in the pipeline showed a downward trend, the flow pattern did not change at this time. 


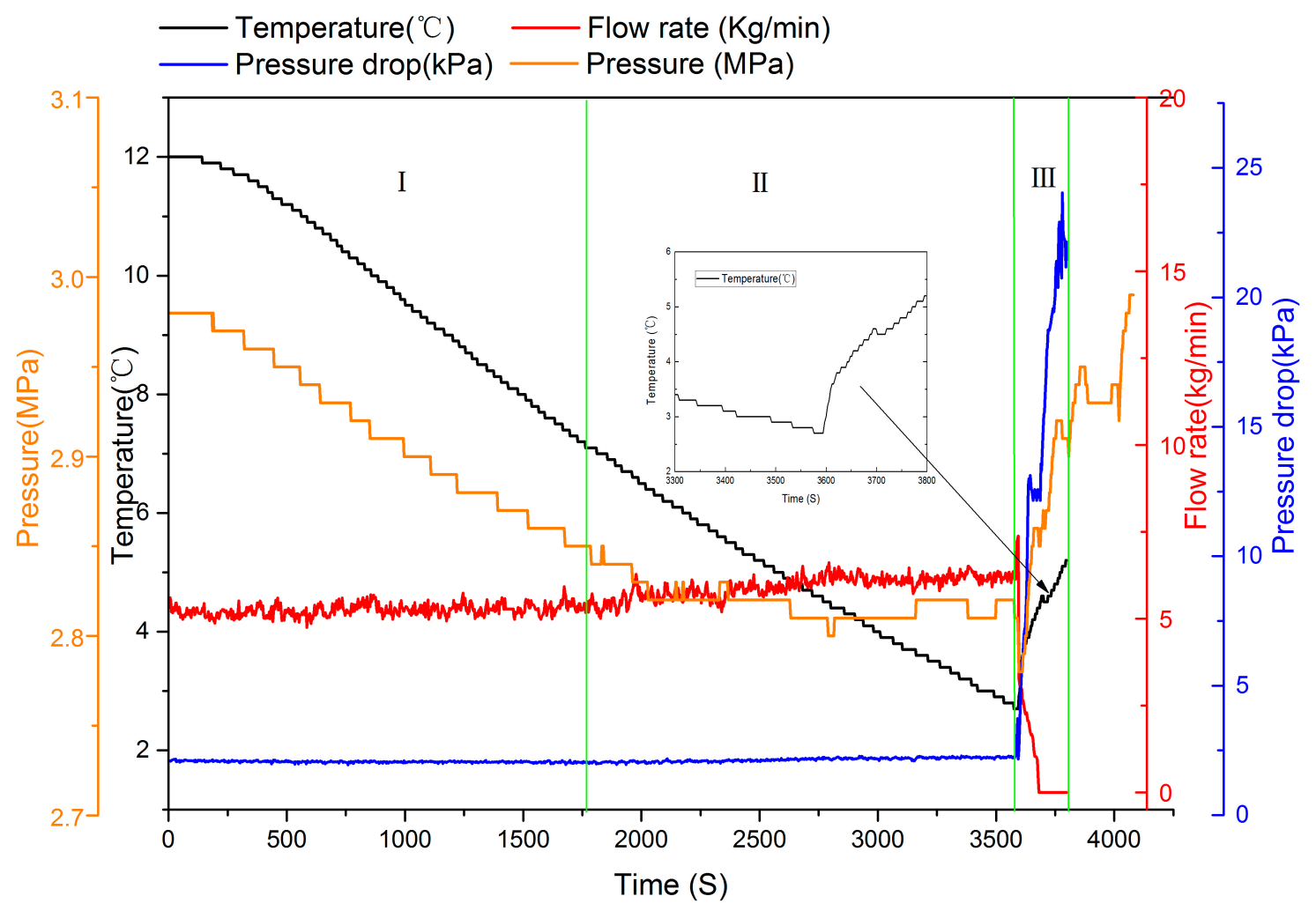

Figure 3. At a pressure of $3 \mathrm{MPa}$, a temperature of $1.5^{\circ} \mathrm{C}$ and a water cut of $60 \%$; the temperature, flow rate and pressure drop with time.

Stage II is the stage of induction period. In this stage, as the temperature reaches the phase equilibrium temperature, the fluid temperature keeps decreasing, but still no hydrate particles appear in the flow loop. When the temperature in the system suddenly rises, indicating that a large amount of hydrate is formed in the loop, the time elapsed in this stage is called the induction stage [22,23]. From group B in Figure 4, a large amount of hydrate particles appear at this time, and the flow is hindered. It can be clearly seen that the hydrate particles flow in the pipe, and a hydrate layer appears at the wall of the pipe, enveloping some oil droplets which do not emulsify, and the slurry flows slowly in the pipe. As the hydrate particles in the pipeline are still forming, growing and aggregating, the viscosity increases and the effective flow area in the pipe is decreased.

Stage III is the stage of slurry flow to the plug. In this stage, with the large amount of hydrate particles forming, the gas-liquid-solid multiphase flow occurs in the flow loop, the differential pressure in the pipeline increases continuously, and the flow rate rapidly decreases. When the lift of the pump is lower than the friction, the differential pressure continues to increase at this time, the flow rate is reduced to zero, and the pipeline is plugged. From group C in Figure 4, the hydrate particles continuously deposit and aggregate at the bottom of the pipe. The viscosity increases and the flow area decreases, resulting in an increase in the flow resistance of the pipe, and ultimately blocking the pipe.

Through the formation and plug processes of hydrates with different water cuts in Figure 4, it can be found that most hydrates start to form at the gas-liquid interface, and a hydrate layer accumulates at the wall of the pipe. The flow at the bottom is in the form of a hydrate slurry, and gradually forms a large aggregate, which affects the fluidity of the pipe and causes plugging. It can be concluded from Group $C$ that the more water cut in the pipeline, the higher volume fraction of hydrates. This volume fraction can also be proved by Figure 5 . 
A
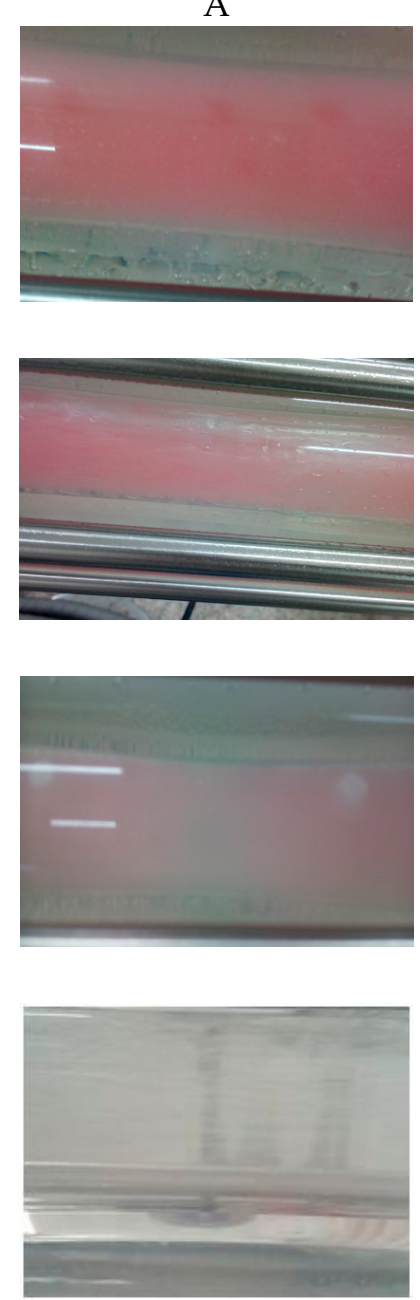

B

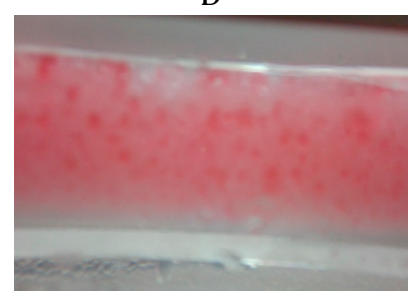

$60 \%$ water cut

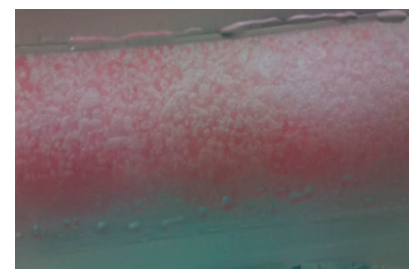

$80 \%$ water cut

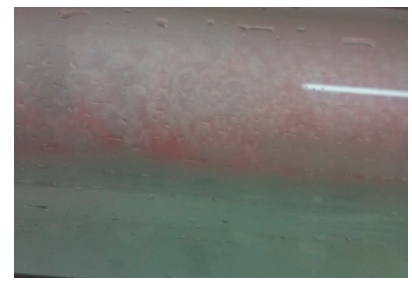

$95 \%$ water cut

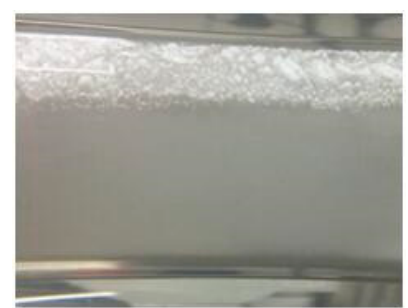

$100 \%$ water cut
C
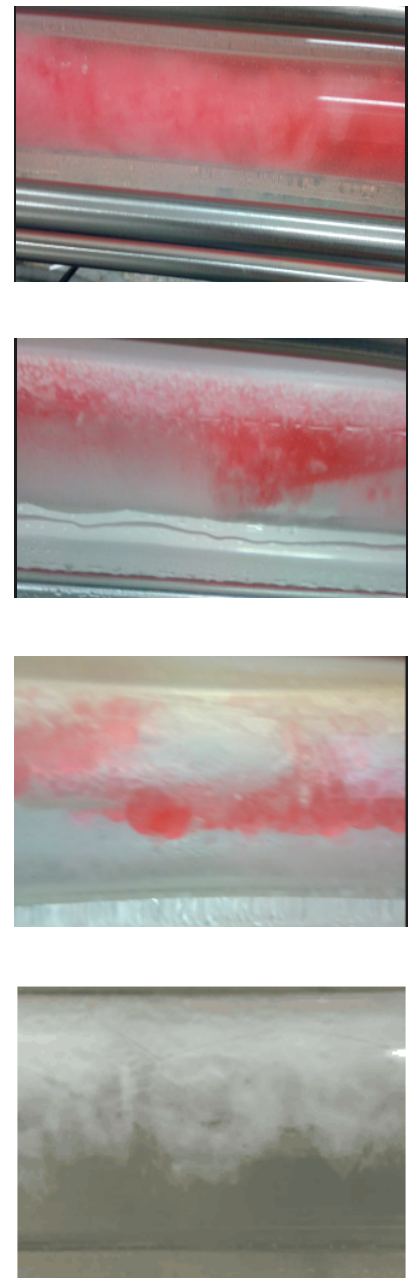

Figure 4. Flow pattern in the pipeline at different water cuts.

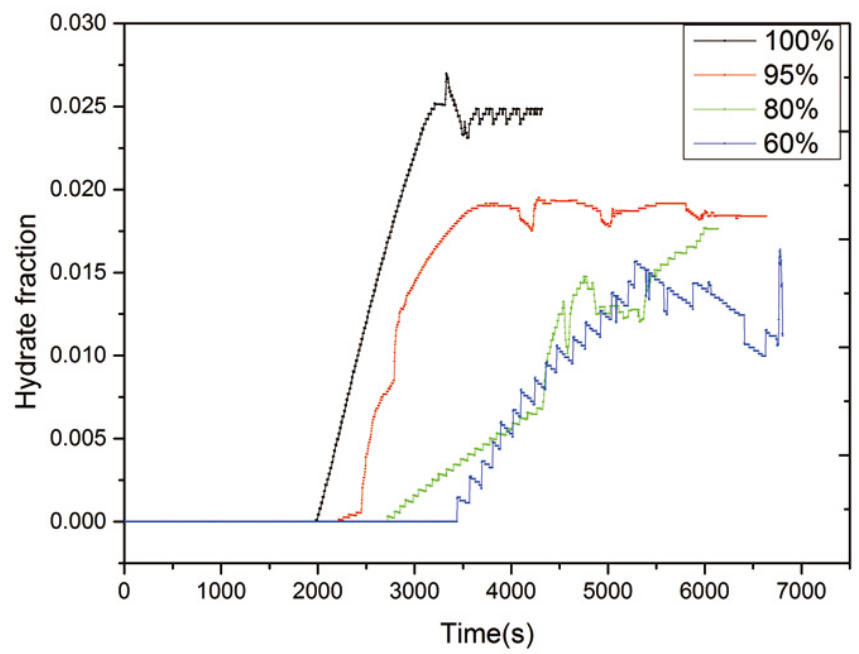

Figure 5. Curve of hydrate volume fraction with time at different water cuts. 


\subsection{Effect of Water Cut on Induction Time of Hydrate Formation}

Hydrate formation includes nucleation and growth processes. When the hydrate nucleus reaches a critical size, the nucleation process ends and the growth stage starts. The induction time is a key factor in this process [24]. The induction time is used as a measure to maintain the sub-stable nature of the supersaturated system $[1,25]$. Based on the macroscopic concept, the time point at which the temperature and pressure reach the three-phase equilibrium point of the system is the initial point $\left(T_{S}\right)$ of the induction time, and the point at which the temperature suddenly rises when a large amount of hydrate starts to form is the end point of the hydrate induction period $\left(T_{e}\right)$. The time difference between these two points is defined as the induction time [26]. The hydrate induction time, $T_{\text {ind }}$, is defined as:

$$
T_{\text {ind }}=T_{e}-T_{S}
$$

Since each test has the same operating condition (the initial temperature, pressure, liquid holding and flow rate), all experiments will have the same point of starting hydrate phase equilibrium. As showed in Figure 6, the hydrate induction time in various systems can be obtained according to the temperature change curve during the experiment. The induction times were $891 \mathrm{~s}, 1020 \mathrm{~s}, 1364 \mathrm{~s}$, and $2252 \mathrm{~s}$ for the conditions with water cuts of $100 \%, 95 \%, 80 \%$, and $60 \%$, respectively. Moreover, it can be observed that the induction time shows a tendency to shorten as the water cut increases.

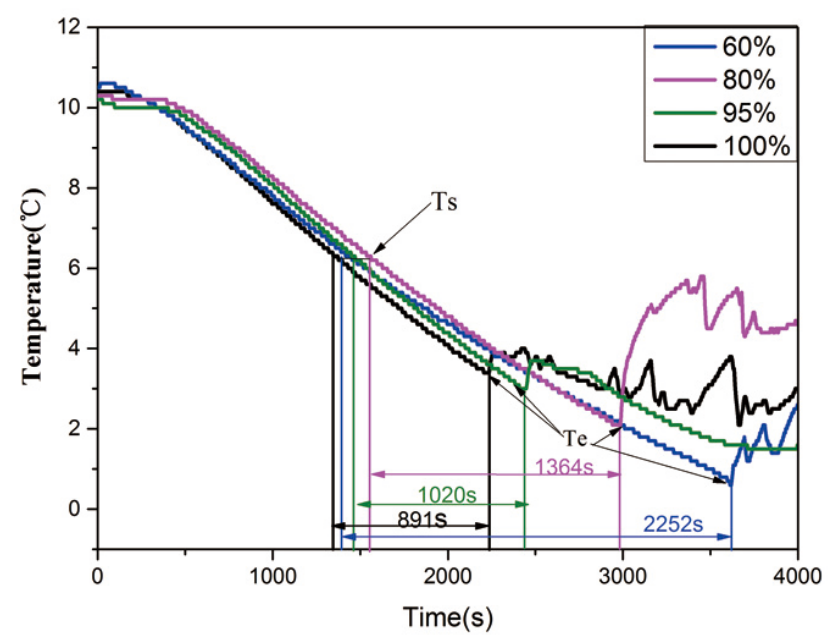

Figure 6. When the initial pressure, temperature, liquid holdup, and flow rate are constant, the hydrate induction time changes under different water cuts.

The plausible explanation of the above phenomenon can be given as follows. In the $100 \%$ water cut system, the gas dissolves into the water and bubbles are also dispersed in the water. Compared with oil-containing systems, the contact opportunities of water and gas in pure water systems are larger, hence the rate of nucleation and aggregation increases. Therefore, the pure water system has a shorter induction time than the oil-containing system. In partially dispersed system, since experiments have not been emulsified, it will form unsteady water-oil and oil-water emulsion in the pipeline. The pipeline has some oil dispersed in the mixture fluid. Further, the surface area of the oil-water interface increases, and the distance between these two phases decreases, which creates a larger contact area between the droplets and $\mathrm{CO}_{2}$ and increases the contact chance of the phases. Therefore, the probability of nucleation increases, and the rate of re-generation of crystal increases. As the growth of the hydrate crystal increased, the induction time was observed to shorten macroscopically. This phenomenon is consistent with the experimental results of Guo's study [27] on the formation of hydrates in methane in oil-containing systems. As the water cuts increase, the induction time decreases. 


\subsection{Effect of Water Cuts on Hydrate Volume Fraction}

The amount of hydrate particles produced in the pipeline affect the fluidity of the slurry in the pipeline. When the amount of hydrate formation is small, the pipe has less possibility to be plugged, and the fluid will carry the hydrate particles flowing fast. When the hydrate volume fraction reaches a certain level, the rheological properties of the pipe fluid will change, leading to plug. Therefore, the volume fraction of the hydrate is an indicator of the safety of the pipe flow. In this paper, the gas solubility model based on the equation of state and particle interaction theory proposed by Duan [28] is used to calculate the dissolved amount of $\mathrm{CO}_{2}$ in water. In this work, the calculation of the hydrate fraction can be performed by the following formula.

The total gas consumption, $\Delta n(\mathrm{~mol})$, was calculated from Equation (2)

$$
\Delta n_{c o_{2}}=\left(\frac{P_{0} V_{0}}{Z_{0} T_{0}}-\frac{P_{t} V_{t}}{Z_{t} T_{t}}\right) \frac{1}{R}
$$

where $V$ is the volume of $\mathrm{CO}_{2}$ in the gas phase; $P$ is the pressure in the pipeline; $T_{0}$ and $T_{t}$ are the temperatures in the pipeline at the beginning of the experiment and at any time of experiment. $Z$ is the gas compression factor, calculated from the PR equation of state [29]; and $R$ is the molar gas constant.

The hydrate volume can be calculated by:

$$
V_{\text {hydrate }(t)}=M \times \Delta n_{\mathrm{CO}_{2}} \times v_{w}^{M T}
$$

where $M$ is the number of hydration, $\Delta n_{\mathrm{CO}_{2}}$ is the gas consumption, and $v_{W}^{M T}$ is the molar volume of $\mathrm{CO}_{2}[30]$, which can be calculated by:

$$
v_{w}^{M T}=\left(11.835+2.217 \times 10^{-5} T+2.242 \times 10^{-6} T^{2}\right)^{3} \times \frac{10^{-30} N_{A}}{46}-8.006 \times 10^{-9} \mathrm{P}+5.448 \times 10^{-12} P^{2}
$$

where $N_{A}$ is the Avogadro constant, $T$ is the temperature of reaction system, and $P$ is the pressure of the reaction system.

The hydrate volume fraction in the pipeline can be calculated by:

$$
\phi_{\text {hyd }}=\frac{V_{\text {hyd }}}{V_{\text {total }}}
$$

where $\phi_{\text {hyd }}$ is the hydrate volume fraction, $V_{\text {hyd }}$ is the hydrate volume, and $V_{\text {total }}$ is $9 \mathrm{~L}$.

The hydrate volume fraction can be calculated by Equation (5), as shown in Figure 5. It can be observed that under the condition that the liquid holding capacity of the pipeline is constant, the volume fraction of the solid phase of hydrates increases as the water cuts increases. In the pure water system, the hydrate volume fraction is the largest at $0.025 \%$, while for the water cut of $60 \%$, the volume fraction is the smallest at $0.015 \%$. Firstly, due to the high water cuts system, the hydrate nucleation and growth have more nucleation sites. Secondly, the larger the water cuts, the larger the oil-water contact area, and the nucleation rate at the gas-water interface will also increase, resulting in an increase in the solid hydrate phase volume fraction as the water cuts increases. It can be seen from Figure 6 that the volume fraction of hydrate particles is small. There are several reasons for this. (1) the gas storage capacity of hydrate is too small; (2) the gas phase space in the pipeline is too small, and the subsequent driving force of hydrate formation is weakened. It can be seen from the comparison of Figures 4 and 6 that the calculated hydrate volume fraction is much lower than that observed, which is due to the error caused by the aggregation of the hydrates.

\subsection{Effect of Water Cuts on the Friction of the Pipeline}

Flow resistance is an important parameter for the flow ability of the reaction tube. Through the study of flow resistance, the main factors that produce resistance in the process of hydrate formation can be defined more clearly, which provides a theoretical basis for ensuring deep-sea oil and gas 
transportation. According to the Darcy-Weisbach formula, the friction and pressure drop during pipe transfer is as follows:

$$
\Delta P=f \frac{L}{R} \rho U^{2}
$$

Therefore, the friction coefficient can be expressed as:

$$
f=\Delta P \frac{R}{L \rho U^{2}}
$$

where $f$ is the change in pipe friction, $\Delta P$ the pressure drop, $L$ is the pipe length, $R$ is the pipe inner diameter, $\rho$ the fluid density, and $U$ is the fluid flow rate.

Figure 7 shows the typical temperature, pressure drop, flow rate, and friction versus time. It can be seen that when a large amount of hydrates form, the flow rate of the system suddenly drops, the pressure drop suddenly rises by about $7.5 \mathrm{kPa}$, and the friction coefficient also suddenly increases. This suggests that the formation of hydrate particles increases the flow resistance and the flow rate is lowered. However, the increase in water cuts will increase the volume fraction of hydrate, which will further enhance the frictional resistance during the process of slurry flow. It can be observed in Figure 8 that the larger the water cuts, the earlier the resistance coefficient is abruptly changed. This is because as the water cuts increases, the induction time is shortened. The formation of hydrates increases the viscosity of the system, resulting in an increase in the resistance coefficient. As the water cuts increase, the absolute coefficient of resistance increases due to the increase in the volume fraction of the hydrate. Due to the instability of the pump and the simultaneous occurrence of hydrate formation and decomposition, the friction coefficient fluctuates greatly, and the resistance coefficient shows a decreasing trend. Therefore, the above resistance coefficient changes can be explained with the following two factors: (1) The viscosity and friction coefficient increase due to the formation of hydrate particles; (2) the coefficient of friction increases due to the collision of hydrate particles with the surface of the tube wall, as well as the agglomeration and deposition of hydrates [31].

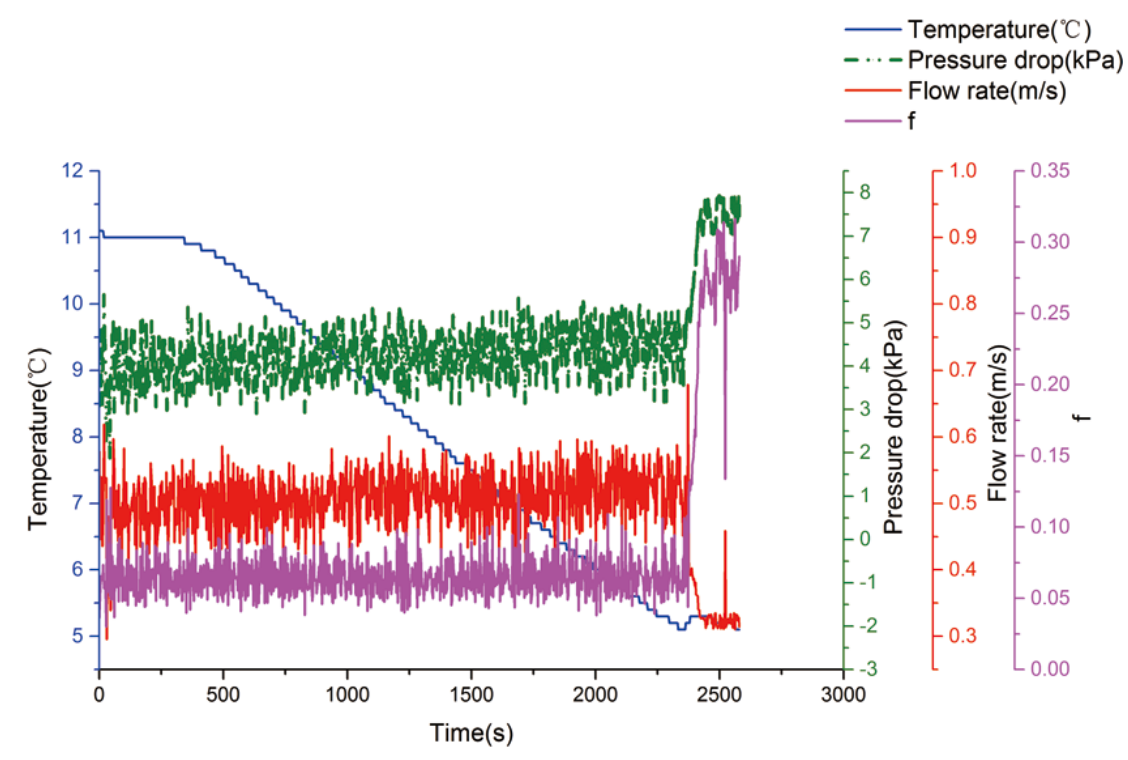

Figure 7. Temperature, flow rate, differential pressure and friction versus time for a water cut of $60 \%$. 


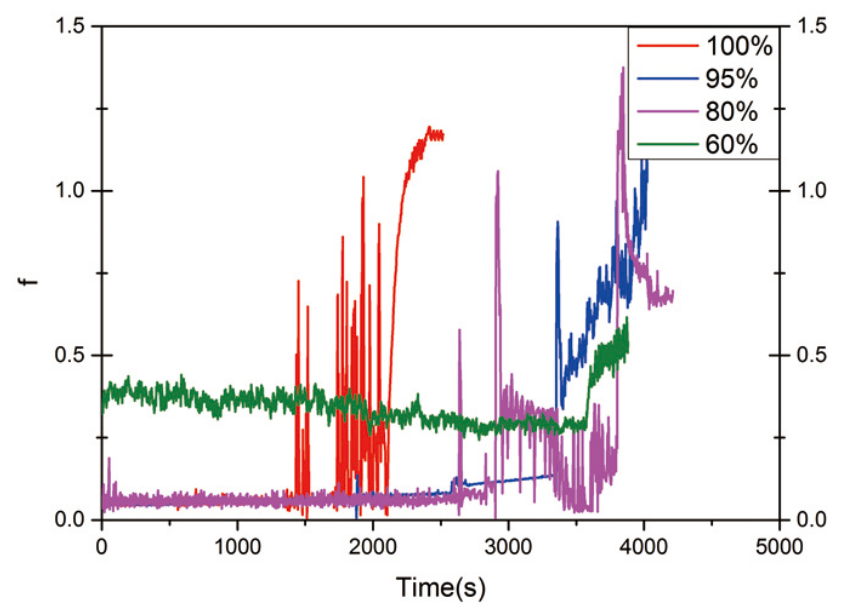

Figure 8. Variation of frictional resistance coefficient in pipelines with different water cut.

\subsection{Effect of Water Cuts on the Safe Flow Time of Hydrate Slurry Flow}

With the continuous deepening of hydrate slurry technology, ensuring the safe transport of slurry is the basis of hydrate plug research. Therefore, the slurry flow time is an important parameter for the safe operation of the pipeline. Figure 9 shows a plot of differential pressure and flow over time for a typical hydrate slurry flow. We can find that after a large amount of hydrates form, the flow rate decreases. Since the hydrate formation is a random phenomenon, the differential pressure fluctuates greatly, and the differential pressure increases but the amplitude is small. As the hydrate particles are still forming, the mutual aggregation between the particles is also enhanced. The velocity and differential pressure fluctuate at 2650s due to the influence of hydrate formation on the stability of pipeline flow. With the aggregation and growth of hydrate particles, the flow rate decreases, resulting in blockage. The flow time of the slurry from generation to plug was $786 \mathrm{~s}$. As the accumulation of the hydrate particles and the deposition layer continue to build up, the frictional resistance continues to increase. It can be observed that the flow rate is the dominant factor at this time, and the decrease of the flow rate causes the continuous reduction of the differential pressure. When the sediment layer and the aggregate reach a certain level, the pipeline is plugged.

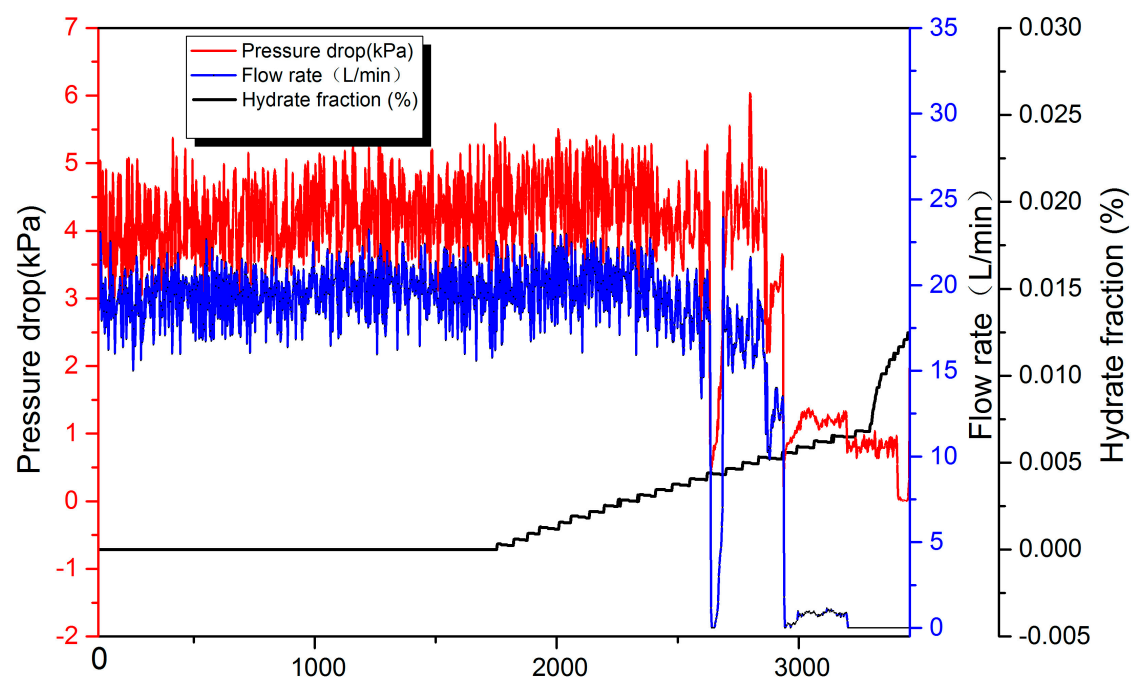

Figure 9. Flow rate and differential pressure versus time for a water cut of $80 \%$.

The flow time of the hydrate slurry at different water cuts is shown in Figure 10. As water cuts increase, the flow time of the hydrate slurry decreases. However, under the condition of high water 
cuts, the flow time of the hydrate does not change much. The reasons for this phenomenon are given in the following: (1) As the water cuts increases, the volume fraction of hydrate increases, the degree of collision and aggregation between the hydrate particles increases, and the volume of aggregates increases, resulting in a decrease in flow time; (2) As the water cuts increases, the contact area of the gas-water increases, and the driving force provided by the gas space after the formation of the hydrate is large, which accelerates the continued formation of the hydrate particles, resulting in a decrease in the fluidity of the slurry, and thus, the flow time is shortened. This can also be verified with the results from Lv et al. [32].

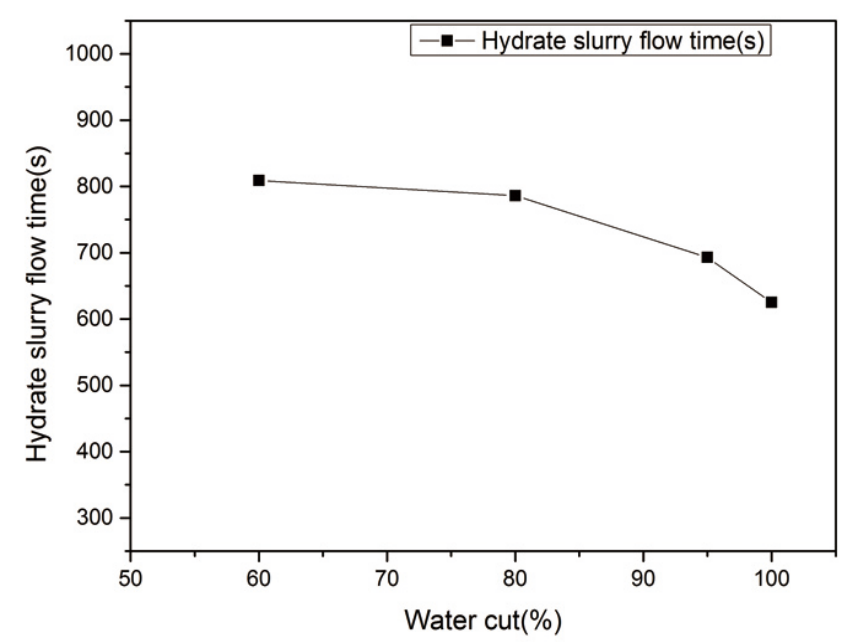

Figure 10. Curve of flow time of hydrate slurry under different water cut.

\subsection{Plugging Mechanism of Hydrate in Medium-High Water Cut}

Regarding the previously discussed experimental phenomena and results, this paper proposes two plugging mechanisms. In different water cuts, it will form two types of emulsions, which are water-in-oil and oil-in-water emulsions in partially dispersed systems. Figure 11 is the schematic of the plugging mechanism for the medium water cuts case. First, in systems with more oil, oil acts as a continuous phase of dispersed water droplets, forming a water-in-oil emulsion. As the temperature continuously decreases to the critical value, the hydrate in the emulsion begins to form due to the presence of saturated dissolved gas in the emulsion. At the same time, some hydrate particles form at the oil-water interface as well. Then, as a large amount of hydrate particles are generated, the probability for collision increases correspondingly [33]. Due to the formation of an unstable water-in-oil emulsion in a medium water system, the mutual aggregation between the hydrate particles in the water-in-oil emulsion is reduced. Thus, they will suspend the fluid and flow in the form of a single small particle. Finally, as the hydrate particles in the aqueous phase continue to aggregate, the increase of the viscosity reduces the velocity, the shearing effect reduces, and the aggregation between the hydrate particles increases as well. As the hydrates deposit, the flow area is lowered. Eventually, the fluid loses fluidity and forms a plug, which touches the safety of the pipe flow.

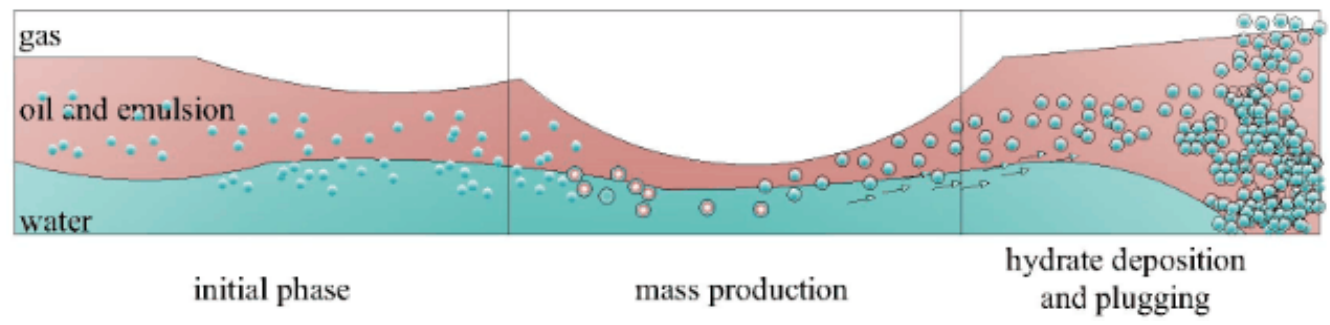

Figure 11. Schematic diagram of plugging mechanism at medium water cut. 
In the case of the high water cut, Figure 12 shows the schematic of the plugging mechanism. First, due to the large amount of water in the pipeline, an oil-in-water emulsion is formed in the upper layer of the water phase, and free water droplets also appear in the oil phase. Then, when the temperature and pressure of the system reach a desired condition, the hydrate starts to form at the oil-water interface, and some form at the gas-water interface. Since the initial pressure is small, the hydrate hole occupancy rate is insignificant, the formed hydrate particle density is unimportant, and the hydrate particles float in the proximity of the pipe wall. The hydrate formed by the oil-in-water emulsion has a strong agglomeration effect, and an aggregate layer is formed in the upper part of the pipe. In addition, a part of the oil phase is fixed in the middle of the hydrate aggregate, as shown in group C in Figure 4. Finally, as the hydrate particles collide with each other, accumulate and deposit at the wall ${ }^{18}$, a sedimentary layer and a moving bed form at the lower tube wall. When the moving bed continuously thickens, a fixed bed forms. As the fixed bed continues to grow, the pipeline gets severely plugged.

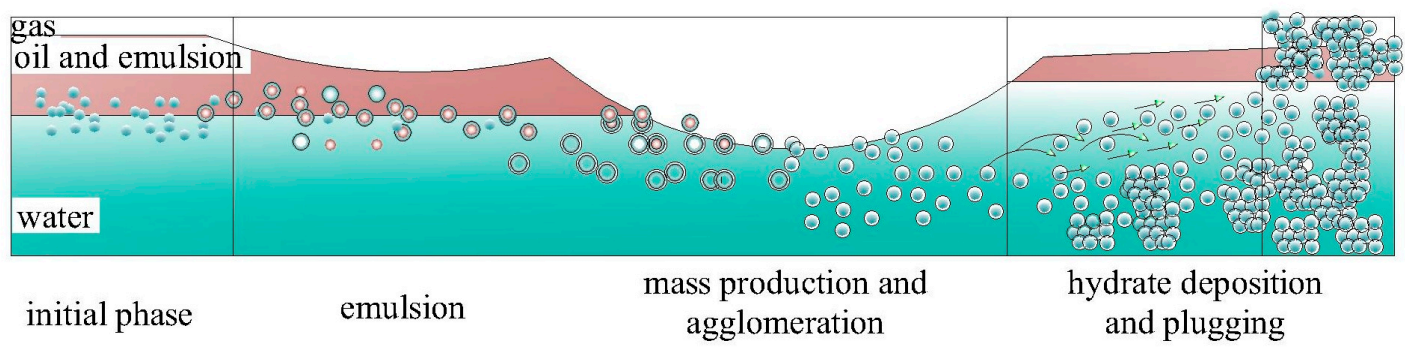

Figure 12. Schematic diagram of plugging mechanism at high water cut.

Notably, plugging conditions in the actual production are usually caused by several plugging mechanisms. The two mechanisms proposed above are just the governing ones during the experiments.

\section{Conclusions}

In this paper, experiments on the formation, flow and plug of hydrates in oil-gas-water three-phase systems with a water cut ranging from $60-100 \%$ were conducted using a high-pressure flow loop. The hydrate formation characteristics, flow characteristics and plugging mechanism were investigated and illustrated in detail. The results presented in this study demonstrated the following conclusions:

(1) When a large amount of hydrates start to form, the temperature of the system rises, the pressure suddenly drops, the pressure drop increases, the flow rate decreases, and the fluidity starts to reduce. When a plug occurs, a portion of the oil phase is held by the hydrate aggregate at the upper tube wall.

(2) As the water cut decreases, the hydrate formation induction time increases, and the final volume fraction of hydrate formation decreases.

(3) As the water cut increases, the friction coefficient increases, and the flow time decreases in the flow of the slurry.

(4) For high water cuts, hydrate nucleation and mass production mainly occur at the gas-water interface. Due to the deposition and aggregation of the hydrates, the change in moving bed to fixed bed will result in plug; for medium water cuts conditions, hydrate nucleation and formation mainly occur at the oil-water interface, and the accumulation of hydrate particles formed by water-in-oil is less. Eventually, it will get plugged due to the increase in viscosity and the accumulation of the deposits.

(5) Moreover, the degree of aggregation among hydrate particles in low water cut systems is less than that in high water cut systems. 
Author Contributions: Writing thesis \& Overall design, S.Z. (Shidong Zhou); Writing an experiment, X.C.; Doing experiments, C.H.; Mechanism analysis, S.W.; Protocol design, S.Z. (Shuhua Zhao); Data Processing, X.L.; Writing-Review \& Editing S.Z. (Shidong Zhou) and X.C.

Funding: This work was supported by [Petro China Innovation Foundation] grant number [2016D-5007-0607], [the National Natural Science Foundation of China grant number [51574045], [National Science Foundation for Young Scientists of China] grant number [Grant No. 51804046], [Petro China Innovation Foundation] grant number [Grant No. 2018D-5007-0602] and [Natural Science ResearceProject of Jiangsu colleges and Universities] grant number [Grant No. 18KJB440001].

Conflicts of Interest: The authors declare no conflict of interest.

\section{References}

1. Zhou, S.; Yan, H.; Su, D.; Navaneethakannan, S.; Chi, Y. Investigation on the kinetics of carbon dioxide hydrate formation using flow loop testing. J. Nat. Gas Sci. Eng. 2018, 49, 385-392. [CrossRef]

2. Zhou, S.; Jiang, K.; Zhao, Y.; Chi, Y.; Wang, S.; Zhang, G. Experimental investigation of $\mathrm{CO}_{2}$ hydrate formation in the water containing graphite nanoparticles and tetra-n-butyl ammonium bromide. J. Chem. Eng. Data 2018, 63, 1-22. [CrossRef]

3. Chi, Y.D. Investigation of Wax Inhibitors on Wax Deposition Based on Flow Loop Testing. M.S. Thesis, University of Tulsa, Tulsa, OK, USA, 2015.

4. Dubey, A.; Chi, Y.; Daraboina, N. Investigating the Performance of Paraffin Inhibitors under Different Operating Conditions. In Proceedings of the SPE Annual Technical Conference and Exhibition, San Antonio, TX, USA, 9-11 October 2017.

5. Chi, Y.; Daraboina, N.; Sarica, C. Investigation of inhibitors efficacy in wax deposition mitigation using a laboratory scale flow loop. AIChE J. 2016, 62, 4131-4139. [CrossRef]

6. Chi, Y.; Daraboina, N.; Sarica, C. Effect of the flow field on the wax deposition and performance of wax inhibitors: Cold finger and flow loop testing. Energy Fuels 2017, 31, 4915-4924. [CrossRef]

7. Daraboina, N.; Chi, Y.; Sarica, C.; Pereyra, E.; Scott, S.L. Effects of High Pressure on the Performance of Existing Two-Phase Flow Models in Wellbores. In Proceedings of the SPE Annual Technical Conference and Exhibition, Dallas, TX, USA, 24-26 September 2018.

8. Ersoy, G.; Sarica, C.; Al-Safran, E.; Zhang, H.Q. Three-phase gas-oil-water flow in undulating pipeline. J. Pet. Sci. Eng. 2017, 156, 468-483. [CrossRef]

9. Chi, Y.; Zhou, S.; Daraboina, N. Experimental Study of Wax Deposition under Two-Phase Gas-Oil Stratified Flow. In Proceedings of the 11th North American Conference on Multiphase Production Technology, Banff, AL, Canada, 6-8 June 2018.

10. Sloan, E.D. Clathrate Hydrates of Natural Gases, 2nd ed.; CRC Press: Boca Raton, FL, USA, 1998, ISBN 978-0849390784.

11. Joshi, S.V.; Grasso, G.A.; Lafond, P.G.; Rao, I.; Webb, E.; Zerpa, L.E. Experimental flowloop investigations of gas hydrate formation in high water cut systems. Chem. Eng. Sci. 2013, 97, 198-209. [CrossRef]

12. Zerpa, L.E.; Rao, I.; Aman, Z.M.; Danielson, T.J.; Koh, C.A.; Sloan, E.D. Multiphase flow modeling of gas hydrates with a simple hydrodynamic slug flow model. Chem. Eng. Sci. 2013, 99, 298-304. [CrossRef]

13. Wang, S.L.; Rao, Y.C.; Zhou, S.D.; Li, J.; Miao, W. An experimental study on deepwater natural gas transmission based on the hydrate slurry flow technology. Nat. Gas Ind. 2014, 34, 101-107.

14. Lorenzo, M.D.; Aman, Z.M.; Kozielski, K.; Bruce, W.E.N.; Johns, M.L.; May, E.F. Underinhibited hydrate formation and transport investigated using a single-pass gas-dominated flowloop. Energy Fuels 2014, 28, 7274-7284. [CrossRef]

15. Lorenzo, M.D.; Aman, Z.M.; Soto, G.S.; Johns, M.; Kozielski, K.A.; May, E.F. Hydrate formation in gas-dominant systems using a single-pass flow loop. Energy Fuels 2014, 28, 3043-3052. [CrossRef]

16. Sloan, D.; Koh, C.; Sum, A.K.; Ballard, A.L.; Creek, J.; Eaton, M. Natural Gas Hydrates in Flow Assurance, 1st ed.; Elsevier: Amsterdam, The Netherlands, 2010, ISBN 9781856179454.

17. Chen, J.; Yan, K.L.; Chen, G.J.; Sun, C.Y.; Liu, B.; Ren, N. Insights into the formation mechanism of hydrate plugging in pipelines. Chem. Eng. Sci. 2015, 122, 284-290. [CrossRef]

18. Akhfash, M.; Aman, Z.M.; Ahn, S.Y.; Johns, M.L.; May, E.F. Gas hydrate plug formation in partially-dispersed water-oil systems. Chem. Eng. Sci. 2016, 140, 337-347. [CrossRef] 
19. Ding, L.; Shi, B.; Lv, X.; Liu, Y.; Wu, H.; Wang, W. Investigation of natural gas hydrate slurry flow properties and flow patterns using a high pressure flow loop. Chem. Eng. Sci. 2016, 146, 199-206. [CrossRef]

20. Song, G.C.; Li, Y.X.; Wang, W.C.; Jiang, K.; Ye, X.; Zhao, P.F. Investigation of hydrate plugging in natural gas+diesel oil+water systems using a high-pressure flow loop. Chem. Eng. Sci. 2017, 158, 480-489. [CrossRef]

21. Sandoval, G.A.B.; Soares, E.J.; Thompson, R.L.; Siqueira, R.D.N.; Andrade, R.M.D.; Campos, F. Analysis of $\mathrm{CO}_{2}$ hydrates in crude oils from a rheological point of view. Energy Fuels 2018, 62, 2733-2741. [CrossRef]

22. Chen, H.T.; Kandasamy, S.B. Effect of chloral hydrate on in vivo kcl-induced striatal dopamine release in the rat. Neurochem. Res. 1996, 21, 695-700. [CrossRef] [PubMed]

23. Sun, C.Y.; Chen, G.J.; Yang, L.Y. Interfacial tension of methane + water with surfactant near the hydrate formation conditions. J. Chem. Eng. Data 2004, 49, 1023-1025. [CrossRef]

24. Dai, W.J.; Wang, S.L.; Rao, Y.C.; Lv, X.F.; Liu, B.; Zheng, Y.X. Experiment on anew accelerant-Graphene oxide for accelerating the formation of $\mathrm{CO}_{2}$ hydrate. Nat. Gas Ind. 2016, 36, 83-88.

25. Zhou, S.; Yu, Y.; Wang, S.; Zhang, G.; Li, H. Research progress in the natural gas hydrate formation model under the pipe flow system. Nat. Gas Ind. 2014, 34, 92-98.

26. Sarshar, M.; Esmaeilzadeh, F.; Fathikalajahi, J. Induction time of hydrate formation in a flow loop. Theor. Found. Chem. Eng. 2010, 44, 201-205. [CrossRef]

27. Guo, Y.D. Study on the Formation/Decomposition Process of Hydrate Slurry in Water-in-Oil Emulsion System. Ph.D. Thesis, China University of Petroleum, Beijing, China, 2016.

28. Duan, Z.H.; Wei, Q. Solubility model of gas $\left(\mathrm{CH}_{4}, \mathrm{H}_{2} \mathrm{~S}, \mathrm{CO}_{2}\right.$, etc.) in aqueous solution. Chin. J. Geol. 2011, 85, 1079-1093.

29. Zhou, S.; Yu, X.; Jiang, K.; YU, X.; Bian, H.; Chen, X. Effect of wax crystal precipitation on the kinetic characteristics of hydrate formation. J. Nat. Gas Ind. 2018, 38, 103-109.

30. Klauda, J.B.; Sandler, S.I. A fugacity model for gas hydrate phase equilibria. Ind. Eng. Chem. Res. 2000, 39, 3377-3386. [CrossRef]

31. Shi, B.; Ding, L.; Liu, Y.; Yang, J.; Song, S.; Wu, H. Hydrate slurry flow property in w/o emulsion systems. RSC Adv. 2018, 8, 11436-11445. [CrossRef]

32. Lv, X.; Shi, B.; Ying, W.; Jing, G. Study on gas hydrate formation and hydrate slurry flow in a multiphase transportation system. Energy Fuels 2013, 27, 7294-7302. [CrossRef]

33. Melchuna, A.; Cameirao, A.; Herri, J.M.; Glenat, P. Topological modeling of methane hydrate crystallization from low to high water cut emulsion systems. Fluid Phase Equilib. 2016, 413, 158-169. [CrossRef] 